\title{
Accretion Effects on Disks Around Non-Magnetic Compact Objects
}

\author{
Michele M. Montgomery \\ University of Central Florida, Department of Physics, \\ 4000 University Blvd., Orlando, FL, USA \\ email: montgomery@physics.ucf.edu
}

\begin{abstract}
Accretion disks in compact binaries are thought to sometimes tilt and precess in the retrograde direction as indicated by modulations in light curves and/or signals. Using 3D Smoothed Particle Hydrodynamics and a low mass transfer rate, Montgomery (2012) shows the disk in non-magnetic Cataclysmic Variables tilts naturally after enough time has passed. In that work, twice the fundamental negative superhump signal $2 \nu_{-}$is associated with disk tilt around the line of nodes, gas stream overflow approximately twice per orbital period, and retrograde precession. In this work, we show that after enough additional time has passed in the same simulation, the $4 \nu_{-}$harmonic appears. The decrease in the $2 \nu_{-}$amplitude approximately equals the amplitude of the $4 \nu_{-}$harmonic. We discuss the implications.
\end{abstract}

Keywords. accretion, accretion disks; hydrodynamics; instabilities; methods: numerical; binaries: close; novae, cataclysmic variables

\section{Introduction}

Positive and negative superhumps (PSH and NSH) are modulations with periods either slightly longer or shorter than the orbital period, respectively. PSH indicate that the disk has expanded beyond the inner Lindblad resonance radius (which is near the 3:1 co-rotational resonance radius) and is precessing in the prograde direction via tidal torques (see e.g., Whitehurst 1988). NSH indicate the disk has tilted around the line of nodes and is precessing in the retrograde direction via tidal torques (Montgomery 2012). PSH are associated with a disk in superoutburst, which is an extended state of high luminosity state, and are common to Cataclysmic Variable (CV) Dwarf Novae (DN) SU UMa and Nova-Like (NL) systems. NSH are also associated with CV NLs and DN during quiescence, during some normal outbursts, and now exclusively during a superoutburst (e.g., Patterson et al. (1995), Gao et al. 1999, Pavlenko et al. 2010, Ohshima et al. 2012). NSH (or signals) seem to be ubiquitous in non-magnetic CVs of all periods and states. As signals suggesting disk tilt and retrograde precession are also common in low-mass X-ray binaries, sources that cause these effects may be common among all compact binary systems. Indeed, Montgomery (2009a) shows retrograde precession of non-co-planer accreting disks in compact binaries is due to the same tidal force that causes the tilted, spinning, oblate Earth to precess in the retrograde direction.

Using 3D Smoothed Particle Hydrodynamics, Montgomery (2012) show disks in nonmagnetic CVs having primary mass is $M_{1}=0.6 \mathrm{M}_{\odot}$ and primary-to-secondary mass ratio is $q=0.45$ do eventually tilt around the line of nodes even though mass transfer to the disk is low. No NSH are seen in the simulated light curve. However, twice the fundamental NSH signal $2 \nu_{-}$is well present from 600-1230 orbital periods. Also shown, the disk is in retrograde precession. What is not shown is any harmonics and any effects to the disk. We address this signal and its effect in this work. 

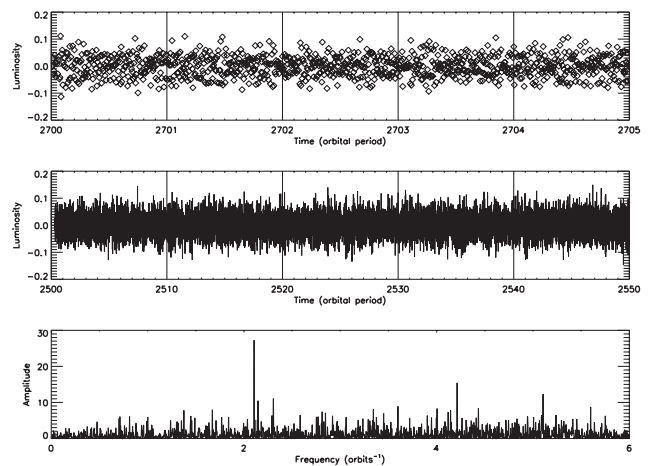

Figure 1. Artificial bolometric light curves (top two panels) and associated Fourier transform (bottom panel). Signals $2 \nu_{-}$and $4 \nu_{-}$are shown in the lowest panel.

\section{Fundamental and Harmonic Signals}

As shown in Montgomery (2012), once the disk tilts, the gas stream flows over the disk rim for approximately one-half an orbital period and then under the disk rim for the latter half orbital period. The net result is approximately two modulations per orbital period in the artificial light curves (Montgomery (2009b)), hence the signal at $2 \nu_{-}=2.106$ orbit $^{-1}$. In addition, if disk tilt is high enough, the NSH signal has a higher amplitude and the gas stream penetrates more deeply into the potential well of the primary, producing stronger NSH modulations in the artificial light curves. As discussed in in Montgomery (2012), the bright spot precesses in the retrograde direction like the First Point of Aries along Earth's equator because, like the Earth, the disk is precessing in the retrograde direction due to tidal torques by the secondary in the binary orbit.

As shown in the figure (Fig. 1), the $4 \nu_{-}=4.211$ orbit $^{-1}$ signal appears at a much later time in the simulation. The procedure to generate the figure is discussed in Montgomery (2012). Once again, no NSH modulations are seen in the light curve, suggesting the disk tilt still remains less than a few degrees. The $2 \nu_{-}$signal strength has decreased in amplitude by an amount that approximately equals the increase in the $4 \nu_{-}$harmonic signal strength. The $2 \nu_{-}$signal strength decrease suggests a decrease in disk tilt: The gas stream overflow now strikes disk annuli that are further from the primary. The $4 \nu_{-}$ signal strength increase suggests the gas stream is spending more time striking the disk rim along the mid-plane during an orbital period. In other words, the bright spot may be modulated more than previously. These suggest the simulation is not yet complete.

\section{References}

Gao, W., Li, Z., Wu, X., Zhang, Z., \& Li, Y. 1999, ApJL, 527, L55

Montgomery, M. M. 2009a, APJ, 705, 603

Montgomery, M. M. 2009b, MNRAS, 394, 1897

Montgomery, M. M. 2012, APJL, 745, L25

Ohshima, T., et al. 2012, PASJ, 64, L3

Pavlenko, E., et al. 2010, in: K. Werner \& T. Rauch (eds.), AIP Conference Series 1273, p. 320

Patterson, J., Jablonski, F., Koen, C., O’Donoghue, D., \& Skillman, D. R. 1995, PASP, 107, 1183

Whitehurst, R. 1988, MNRAS, 232, 35 